\title{
Echinococcus multilocularis vesicular fluid inhibits activation and proliferation of natural killer cells
}

\author{
Anne-Pauline Bellanger ${ }^{1,2}$, Valentine Mougey ${ }^{1}$, Jean-René Pallandre ${ }^{3}$, Houssein Gbaguidi-Haore ${ }^{4}$, Yann Godet ${ }^{3}$ \\ and Laurence Millon ${ }^{1,2}$
}

\author{
${ }^{1}$ Parasitology-Mycology Department, University Hospital, Besançon, France; \\ ${ }^{2}$ Chrono-Environnement CNRS 6249 Research Team, Franche-Comté University, Besançon, France; \\ ${ }^{3}$ INSERM Unit 1098, University of Franche-Comté, Besançon, France; \\ ${ }^{4}$ Infection Control Department, Besançon University Hospital, Besançon, France
}

\begin{abstract}
Alveolar echinococcosis is a severe chronic helminthic disease that mimics slow-growing liver cancer. The immune evasion strategy of Echinococcus multilocularis Leuckart, 1863 remains poorly understood. The aim of this study was to investigate in vitro the impact of E. multilocularis vesicular fluid (Em-VF) on peripheral blood mononuclear cells (PBMC) and on natural killer (NK) cells. PBMC and NK cells were exposed to Em-VF $(1 \mu \mathrm{g} / \mathrm{ml})$ during six days. The effect of $E m$-VF was assessed on CD69, viability and proliferation, and on and transforming growth factor $\beta$ (TGF- $\beta$ ), interferon $\gamma$ (IFN- $\gamma$ ), interleukin 17 (IL-17) and interleukin 10, using flow cytometry and ELISA, respectively. Exposure to Em-VF had no bearing on PBMC's viability, proliferation and expression of CD69. In contrast, higher levels of IL-17 at day three and of TGF- $\beta$ at day six were observed in PBMC supernatant after exposure to Em-VF $(p<0.05$, Wilcoxon signed-rank test). Exposure to Em-VF induced a significant decrease of CD69 expression of NK cells at day three and a significant decrease of proliferation of NK cells at day six $(p<0.05$, Wilcoxon signed-rank test). In contrast, NK cells viability and levels of cytokines did not vary significantly over $E m$-VF stimulation. Exposure to $E m$-VF had a significant bearing on activation and proliferation of NK cells. NK cells may play an important role in the immune response of the host against E. multilocularis.
\end{abstract}

Keywords: NK cells, alveolar echinococcosis, immune response, CD69

Alveolar echinococcosis (AE) is a severe chronic helminthic disease caused by the tapeworm Echinoccocus multilocularis Leuckart, 1863, that mimics slow-growing liver cancer. Experimental studies in infected mice as well as immunologic studies in AE patients have revealed some insight of the complex host-parasite relationship that develops during $\mathrm{AE}$ (Wang and Gottstein 2016). It is now recognised that $\mathrm{AE}$ is characterised by an initial Th1-oriented response followed gradually by a mixed $\mathrm{Th} 1 / \mathrm{Th} 2$ response (Vuitton et al. 2006, Wang and Gottstein 2016). $\mathrm{T}$ cell lineages appear to play a key role in the reorientation of the immune response (Vuitton et al. 2006, Wang and Gottstein 2016). Persistence of E. multilocularis is recognised to be the result of immune tolerance, mainly mediated by T-regulatory cells (Tregs) (Gottstein et al. 2017).

Previous murine AE model described abnormalities observed on crucial innate immune actors such as dendritic cells, during infection: down-regulation of co-stimulary molecules and production of TGF- $\beta$ (Mejri et al. 2011), death mediated by apoptosis (Nono et al. 2012) and abrogation of lymph node cells proliferation (Margos et al.
2011). Natural killer (NK) cells are a type of cytotoxic lymphocytes that are also critical to the innate immune system because they provide rapid responses to harmful cells, acting without prior activation (Vivier et al. 2011). Natural killer T (NKT) cells are a heterogeneous group of $\mathrm{T}$ cells that share properties of both $\mathrm{T}$ cells and NK cells. They constitute a small part of peripheral blood T cells and differs from NK cells (Jerud et al. 2006).

In the present study, we chose to focus on NK cells for two main reasons: (1) their unique ability to recognise stressed cells in the absence of prior activation, allowing for a much faster immune reaction (Vivier et al. 2011), and (2) their recognised role in tumour surveillance, demonstrated in both mice and human models (Moretta et al. 2016, Muenst et al. 2016). NK cells are currently considered as interesting candidates for cancer immunotherapy (Iannello et al. 2016, Moretta et al. 2016, Muenst et al. 2016). Proliferation of E. multilocularis and the development of malignant tumors are both sharing similar features such as local immune evasion, induction of tolerance and disruption of T cell signalling (Wang and Gottstein 2016).

Address for correspondence: A.-P. Bellanger, Parasitology-Mycology Department, University Hospital, 2 boulevard Fleming, Besançon, France. Phone: 33 (0)3 706323 51; Fax: 33 (0)3 706323 64; E-mail: apbellanger@chu-besancon.fr 
Surprisingly, the impact of infection with E. multilocularis on NK cells has received little attention up to now, with only two publications reporting results on these cells (Vuitton et al. 1989, Zhang et al. 2008). This is the reason why, in the present study, we aimed to investigate in vitro the impact of E. multilocularis on NK cells.

\section{MATERIALS AND METHODS}

\section{Echinococcus multilocularis vesicular fluid}

E. multilocularis vesicular fluid (Em-VF), obtained from in vitro-cultured vesicles, was kindly provided by Bruno Gottstein (Institute of Parasitology, Bern University, Bern, Switzerland). Total protein content of Em-VF was measured with the Lowry technique using the DC protein assay (Bio-Rad, Marne La Coquette, France). Em-VF was used for cell stimulation at $1 \mu \mathrm{g} / \mathrm{ml}$ (Bellanger et al. 2015).

\section{Peripheral blood mononuclear cells and preparation of NK cells}

Total blood was obtained from healthy donors using cytapheresis kits (French Blood Institute of Bourgogne Franche-Comté). All donors whose cells were tested were informed and signed an agreement explaining that the remaining blood of their donation would be used for research projects. The 'healthy status' of each donor was carefully checked before each donation by clinicians (clinical examination and interview, biological analysis). Peripheral blood mononuclear cells (PBMC) were isolated by density gradient centrifugation (Ficoll-Paque ${ }^{\mathrm{TM}}$ Premium, Dutscher, Brumath, France). PBMC were washed repeatedly, counted and suspended in RPMI 1640 (Dutscher) supplemented with 10\% heat-inactivated fetal calf serum (iFCS, Dutscher) and $1 \%$ penicillin streptomycin (PS, Dutscher). NK cells were specifically sorted using the Easysep Human Enrichment NK cells kit (Stemcell Technologies, Grenoble, France). Seventeen donors were tested during the study (all were men, mean age $48 \pm 8$ years).

\section{Stimulation protocols}

NK cells were seeded in 96-well plates at $2 \times 10^{5}$ cells/ $200 \mu \mathrm{l} /$ well in RPMI supplemented with $10 \%$ iFCS. NK cells were stimulated with $E m$-VF $(1 \mu \mathrm{g} / \mathrm{ml})$ at day 0 and at day 3. Similarly, PBMC were seeded $2 \times 10^{5}$ cells $/ 200 \mu \mathrm{l} /$ well and stimulated with $E m$-VF $(1 \mu \mathrm{g} / \mathrm{ml})$ at day 0 and at day 3. The dosage of $1 \mu \mathrm{g} / \mathrm{ml}$ of $E m$-VF was selected from a previous work (Bellanger et al. 2015). Non-stimulated PBMC cells (negative control) and EmVF stimulated PBMC cells were both cultivated in the presence of interleukin 2 (IL-2) (30 UI per well). PBMC cells cultivated in the presence of Dynabeads ${ }^{\circledR}$ Human $\mathrm{T}$ activator (Life technologies, Saint Aubin, France) were used as a positive control.

Non-stimulated NK cells (negative control) and Em-VF stimulated NK cells were both cultivated in the presence of IL-2 (100 UI per well). NK cells cultivated in the presence of higher quantity of IL-2 (1,000 UI per well) were used as a positive control.

\section{Flow cytometry analysis}

Sorted NK cells were stained before and after Em-VF exposure with labelled antibodies against $\mathrm{CD} 3\left(\mathrm{PE}-\mathrm{Cy}^{\mathrm{TM}} 7\right)$ and $\mathrm{CD} 56$ (PE) (BD Biosciences Pharmingen, Pont de Claix, France). T cells were stained before and after $E m$-VF exposure with labelled anti- bodies against $\mathrm{CD} 3$ (PE-Cy ${ }^{\mathrm{TM}} 7$ ) and $\mathrm{CD} 4$ (PE) (BD Biosciences Pharmingen). Their activation was assessed via the expression of the differentiation antigen CD69 (FITC) (BD Biosciences), which is expressed shortly after activation and has been shown to trigger NK cell cytolytic activity (Borrego et al. 1999). The impact of Em-VF stimulation on NK cells proliferation was assessed using Cell proliferation Dye eFluor ${ }^{\circledR} 450$ (Affymetrix eBioscience, Paris, France). T cells and NK cells were measured by flow cytometry (BD Canto II) using the FACS DIVA software.

\section{Immunoassays}

Before and after stimulation of NK cells and PBMC with E. multilocularis-VF, supernatants were collected for ELISA testing cytokines. For NK cells, the secretion of two cytokines was measured: interferon $\gamma$ (IFN- $\gamma$ ) (Diaclone, Besançon, France) and transforming growth factor $\beta$ (TGF- $\beta$ ) (Promega, Charbonnières les Bains, France). For PBMC, the secretion of four cytokines was measured: IFN- $\gamma$, IL-10 and IL-17 (Diaclone) and TGF- $\beta$ (Promega). Each sample was measured in duplicate, following the manufacturer's instructions. The TGF- $\beta$ assay used allowed a specific detection of TGF- $\beta_{1}$, with less than $3 \%$ cross-reactivity with other related growth factors (TGF- $\beta_{2}$ and TGF- $\beta_{3}$ ).

\section{Statistical analysis}

Data are presented as means \pm SD from at least ten separate experiments. For comparison of continuous paired data (related samples) the Friedman test followed by the Wilcoxon signedrank test was carried out using the software package Stata v10 (StataCorp LP, College Station, TX, USA). All tests were twotailed, and a $p$ value of less than 0.05 was considered statistically significant.

\section{RESULTS}

\section{NK cell activation in response to $\mathbf{E m - V F}$}

CD69 expression and proliferation for NK cells were gated for CD3 negative CD56 positive cells. A mean decrease of proliferation of $3.5 \%$ was observed between non-stimulated NK cells and Em-VF stimulated cells at day six ( $p<0.05$, Wilcoxon signed-rank test) (Fig. 1A). In the same way, a mean decrease of CD69 expression at day three of $3.3 \%$ was observed between non-stimulated NK cells and Em-VF stimulated cells ( $p<0.05$, Wilcoxon signed-rank test) (Fig. 1A). In contrast, Em-VF stimulation had no bearing on the viability of the NK cells (Fig. 1B).

\section{Activation of $\mathrm{T}$ cells in response to $\mathrm{Em}-\mathrm{VF}$}

CD69 expression and proliferation for T cells were gated for CD3 positive CD4 positive cells. Em-VF stimulation did not significantly modify any parameters investigated for T cells (proliferation, CD69 expression and viability) (Fig. 2).

\section{NK supernatant levels of IFN- $\gamma$ and TGF- $\beta$ in response to $E m-V F$}

Results of ELISA at day three and day six for IFN- $\gamma$ and TGF- $\beta$ secreted by NK cells are presented in Fig. 3; no significant difference was observed at day three and day six between levels of IFN- $\gamma$ and TGF- $\beta$ for non-stimulated NK 

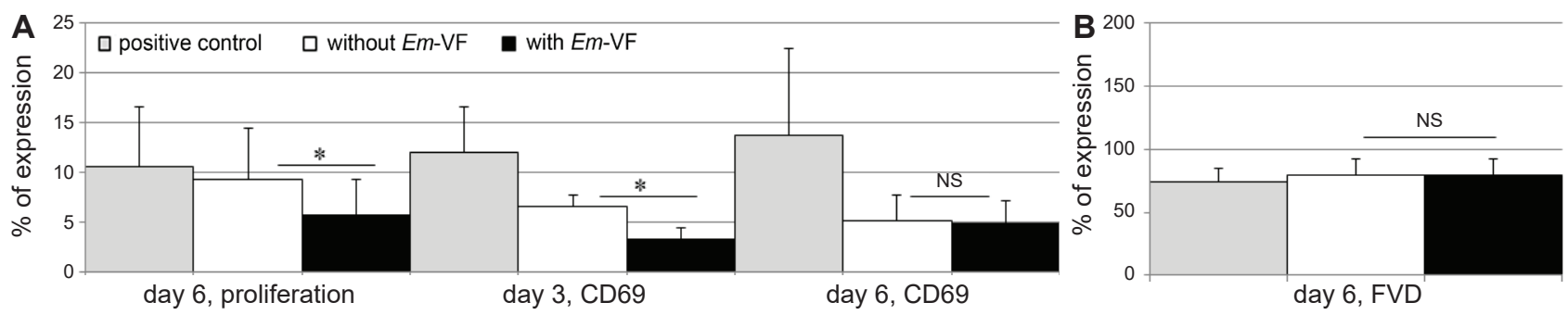

Fig. 1. Natural killer (NK) cells proliferation, activation (A) and viability (B) upon stimulation by Echinococcus multilocularis vesicular fluid $(E m-\mathrm{VF})\left({ }^{*} p<0.05\right.$, Wilcoxon signed-rank test). Non-stimulated NK cells (negative control) and $E m$-VF stimulated NK cells were both cultivated in the presence of Interleukin (IL-2) (100 UI per well). NK cells cultivated in the presence of higher quantity of IL-2 (1,000 UI per well) were used as a positive control. Abbreviations: FVD - fixable viability dye ; NS - non significant.
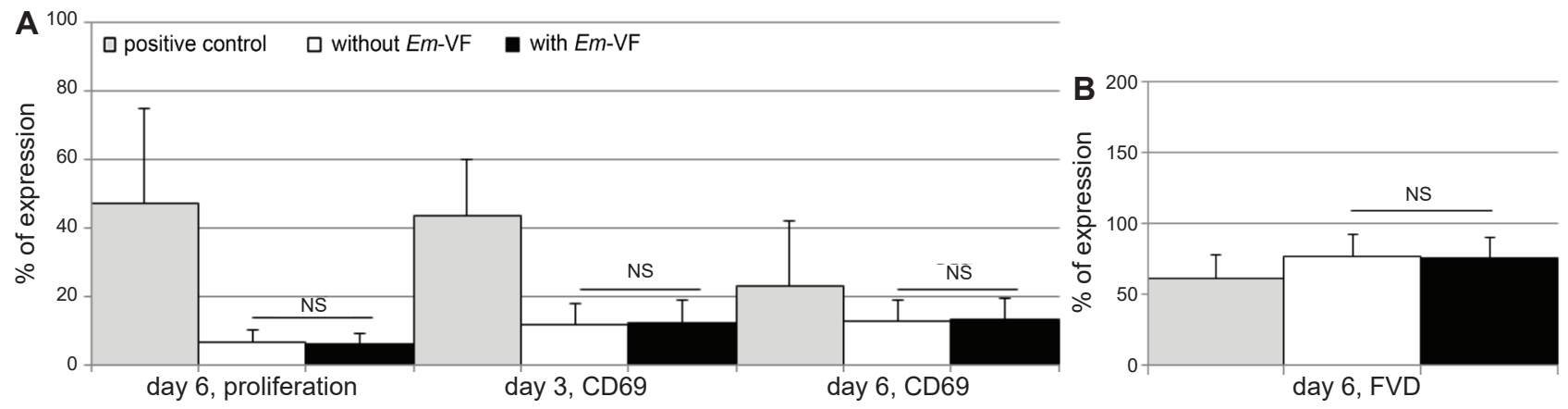

Fig. 2. T cells proliferation, activation (A) and viability (B) upon stimulation by Echinococcus multilocularis vesicular fluid (Em-VF). Non-stimulated cells (negative control) and Em-VF stimulated cells were both cultivated in the presence of interleukin 2 (30 UI per well). Cells cultivated in the presence of Dynabeads were used as a positive control. Abbreviations: FVD - fixable viability dye ; NS - non significant.
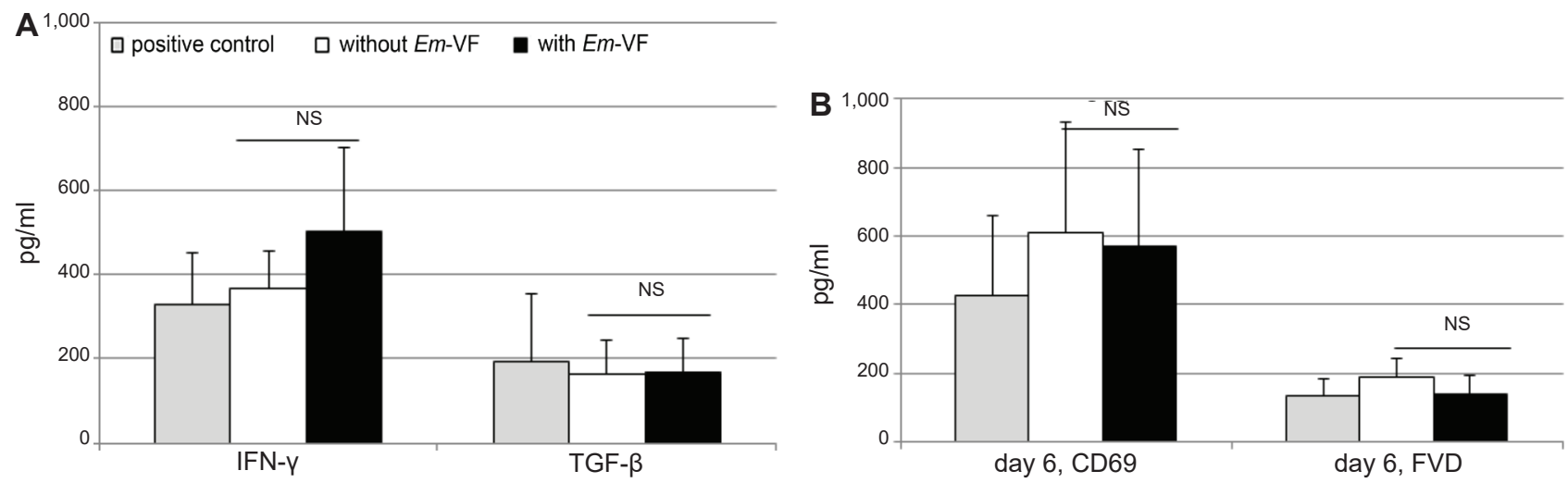

Fig. 3. Levels of interferon $\gamma(\mathrm{IFN}-\gamma)$ and transforming growth factor (TGF- $\beta$ ) released at day three (A) and day six (B) upon stimulation by Echinococcus multilocularis vesicular fluid (Em-VF). No significant differences were observed between non-stimulated natural killer cells (NK) and Em-VF stimulated NK cells ( $p>0.05$, Wilcoxon signed-rank test). Abbreviation: NS - non significant.

cells and Em-VF stimulated NK cells in their respective supernatants.

\section{PBMC supernatant levels of IL-10, IL-17, TGF- $\beta$ and IFN- $\gamma$ in response to $E m-V F$}

Results of ELISA at day three and day six for IFN- $\gamma$, IL-10, IL-17 and TGF- $\beta$ secreted by PBMC are presented in Fig. 4; a significant difference of IL-17 level was observed at day three between non-stimulated PBMC and Em-VF stimulated PBMC $(p<0.05$, Wilcoxon signed-rank test). A significant increase of TGF- $\beta$ secreted by Em-VF stimulated PBMC at day six was also observed $(p<0.05$, Wilcoxon signed-rank test). No significant difference was observed at day three and day six between levels of IFN- $\gamma$ and IL-10 for non-stimulated PBMC and Em-VF stimulated $\mathrm{PBMC}$ in their respective supernatants.

\section{DISCUSSION}

The assays performed showed that exposition to Em-VF induced a significant decrease of the proliferation of NK cells and a similar decrease of activation of marker CD69 of NK cells. In contrast, exposition to Em-VF had no bearing on T cells on viability, proliferation or expression of the activation marker CD69.

In the past 30 years, many experimental studies have investigated the tolerance mechanisms involved in the 


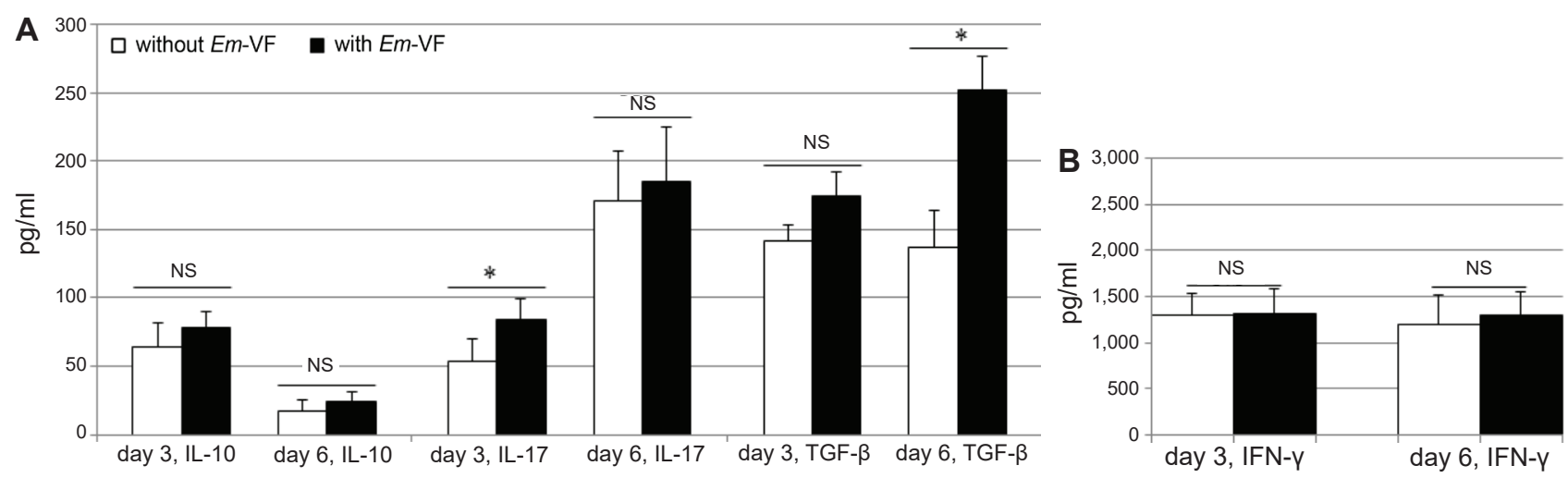

Fig. 4. Levels of interleukin 10 (IL-10), interleukin 17 (IL-17), transforming growth factor $\beta$ (TGF- $\beta$ ) (A) and interferon $\gamma$ (IFN- $\gamma$ ) (B) released at day three and day six upon stimulation by Echinococcus multilocularis vesicular fluid (Em-VF) $(* p<0.05$, Wilcoxon signed-rank test). A significant increase of IL-17 at day three and TGF- $\beta$ at day six was observed. No significant differences were observed between non-stimulated peripheral blood mononuclear cells (PBMC) and Em-VF stimulated PBMC cells for IL-10 and IFN- $\gamma$.

Echinococcus multilocularis/host relationship to understand how the parasite escapes the immune host response. It is now recognised that experimental murine AE is immunologically characterised by a Th1-oriented response at the early stage of infection which is gradually replaced by a mixed Th1/Th2 response during the chronic phase of AE (Vuitton et al. 2006, Wang and Gottstein 2016). The T cell lineages are considered as key actors in the re-orientation of the host immune response (Vuitton et al. 2006, Wang and Gottstein 2016).

The secretion of TGF- $\beta$ and IL-17, observed in the present study after exposition of PBMC to the parasite in vitro, is in accordance with other previous studies (Wang et al. 2014, Bellanger et al. 2015).

Surprisingly, very little is known about the role of NK cells during AE. Only two studies investigated whether NK cells may play a role in AE immune regulatory processes. Both publications support that an inhibition of NK cell homing to the lesions and/or their activation could be involved at advanced stages of metacestode settlement in the liver. Vuitton et al. (1989) measured a lower NK cell cytotoxicity in PBMC of patients with $\mathrm{AE}$ in comparison with patients with non-parasitic biliary disease (Vuitton et al. 1989). Zhang et al. (2008) observed a low number of NK cells associated with the lack of expression of NKG2D on $\mathrm{CD}^{+} \mathrm{T}$ lymphocytes in liver sections of patients with AE (Zhang et al. 2008). The present in vitro study suggests that NK cells are impacted at the early stage of the host/ parasite interactions and that they should be targeted by more studies.

The range of decreases for both proliferation and CD69 expression is low $(\approx 3 \%)$, but this range of percentage variations is not unusual in flow cytometry assays. For ex- ample, increased expression of CD69 has been recently reported and ranged from 2 to $10 \%$ in a murine model of pancreatic adenocarcinoma (Azad et al. 2017). In fact, description of inhibitory mechanism is quite rare: most of the publications on viruses, fungi and parasites are describing upregulation and overexpression of markers or cytokines. The induction of inhibitory mechanism is specific of E. multilocularis, especially its ability to impair the maturation of dendritic cells, as previously suggested by other studies (Mejri et al. 2011, Bellanger et al. 2015).

The present study suggests again that NK cells should be targeted by more assays, both in vitro and in vivo, investigating the complex immune processes taking place during AE. More specifically other activation surface markers specific to NK cells such as NKG2D, NKp44, NKp46 and NKp30 should be investigated. Among them NKG2D seems to be of particular interest as the lack of its expression at advanced stages of metacestode settling was previously reported (Zhang et al. 2008) and could be the sign of an additional mechanism of immune evasion that should be studied in vitro and in vivo.

Impairment of functions of NK cells could be an important factor in the immune evasion strategy displayed by E. multilocularis for its survival in the host. Future in vitro and in vivo studies on the complex relationship between E. multilocularis and the host should focus more on NK cells.

Acknowledgements. We thank Pamela Albert for her editorial assistance. We thank Bruno Gottstein (Institute of Parasitology, Bern, Switzerland) who provided the E. multilocularis vesicular fluid. This study was supported financially by the Immunology Cancer Biotherapy network.

\section{REFERENCES}

Azad A., Yin Lim S., D’Costa Z., Jones K., Diana A., Sansom O.J., Kruger P., Liu S., McKenna W.G., Dushek O., MusCHEL R.J., FoKas E. 2017: PD-L1 blockade enhances response of pancreatic ductal adenocarcinoma to radiotherapy. EMBO Mol. Med. 9: 167-180.
Bellanger A.P., Pallandre J.R., Gbaguidi-Haore H., KnapP J., Malézieux N., Lignon T., Borg C., Millon L. 2015: Investigating the impact of Echinococcus multilocularis vesicular fluid on human cells from healthy blood donors. J. Immunol. Methods 417: 52-59. 
Borrego F., Robertson M.J., Ritz J., Peña J., Solana R. 1999: CD69 is a stimulatory receptor for natural killer cell and its cytotoxic effect is blocked by CD94 inhibitory receptor. Immunology 97: 159-165.

Gottstein B., Soboslay P., Ortona E., Wang J., SiracusaNo A., Vuitton D.A. 2017: Immunology of alveolar and cystic echinococcosis (AE and CE). Adv. Parasitol. 96: 1-54.

Iannello A., Thompson T.W., Ardolino M., Marcus A., RAULET D.H. 2016: Immunosurveillance and immunotherapy of tumors by innate immune cells. Curr. Opin. Immunol. 38: $52-58$.

Jerud, E.S., Bricard G., Porcelli S.A. 2006: Natural killer T cells: roles in tumor immunosurveillance and tolerance. Transfus. Med. Hemother. 33: 18-36.

Margos M.C., Grandgirard D., Leib S., Gottstein B. 2011: In vitro induction of lymph node cell proliferation by mouse bone marrow dendritic cells following stimulation with different Echinococcus multilocularis antigens. J. Helminthol. 85: 128-137.

Mejri N., Müller J., Gottstein B. 2011: Intraperitoneal murine Echinococcus multilocularis infection induces differentiation of TGF- $\beta$-expressing DCs that remain immature. Parasite Immunol. 33: 471-482.

Moretta L., Pietra G., Vacca P., Pende D., Moretta F., Bertaina A., Mingari M.C., Locatelli F., Moretta A. 2016 : Human NK cells: From surface receptors to clinical applications. Immunol Lett. 178: 15-19.

Muenst S., Läubli H., Soysal S.D., Zippelius A., Tzankov A., Hoeller S. 2016: The immune system and cancer evasion strategies: therapeutic concepts. J. Intern. Med. 279: 541-562.

Nono J.K., Pletinckx K., Lutz M.B., Brehm K. 2012: Excretory/secretory-products of Echinococcus multilocularis larvae induce apoptosis and tolerogenic properties in dendritic cells in vitro. PLoS Negl. Trop. Dis. 6: e1516.

Vivier E., Raulet D.H., Moretta A., Caligiuri M.A., ZitvoGel L., Lanier L.L., Yokoyama W.M., Ugolini, S. 2011: Innate or adaptive immunity? The example of natural killer cells. Science 331: 44-49.

Vuitton D.A., Bresson-Hadni S., Laroche L., Kaiserlian D., Guerret-Stocker S., Bresson J.L., Gillet M. 1989: Cellular immune response in Echinococcus multilocularis infection in humans. II. Natural killer cell activity and cell subpopulations in the blood and in the periparasitic granuloma of patients with alveolar echinococcosis. Clin. Exp. Immunol. 78: 67-74.

Vuitton D.A., Zhang S.L., Yang Y., Godot V., Beurton I., Mantion G., Bresson-Hadni S. 2006: Survival strategy of Echinococcus multilocularis in the human host. Parasitol. Int. 55: S51-S55.

Wang J., GotTstein B. 2016: Immunoregulation in larval Echinococcus multilocularis infection. Parasite Immunol. 38: 182-192.

Wang J., Lin R., Zhang W., Li L., Gottstein B., BlagoskLonov O., Lü G., Zhang C., Lu X., Vuitton D.A., Wen H. 2014: Transcriptional profiles of cytokine/chemokine factors of immune cell-homing to the parasitic lesions: a comprehensive one-year course study in the liver of E. multilocularis-infected mice. PLoS ONE 9: e91638.

Zhang S., Hüe S., Sène D., Penfornis A., Bresson-Hadni S., Kantelip B., Caillat-Zucman S., Vuitton D.A. 2008: Expression of major histocompatibility complex class I chain-related molecule A, NKG2D, and transforming growth factor-beta in the liver of humans with alveolar echinococcosis: new actors in the tolerance to parasites? J. Infect. Dis. 197: 1341-1349.

Cite this article as: Bellanger A.-P., Mougey V., Pallandre J.-R., Gbaguidi-Haore H., Godet Y., Millon L. 2017: Echinococcus multilocularis vesicular fluid inhibits activation and proliferation of natural killer cells. Folia Parasitol. 64: 029. 